\title{
The Role of Information Update in Flow Control
}

\author{
Eitan Altman ${ }^{\star 1}$, Tamer Başar ${ }^{\star \star 2}$, and Naceur Malouch ${ }^{3}$ \\ 1 INRIA, BP 93, 06902 Sophia Antipolis Cedex, FRANCE \\ 2 University of Illinois, 1308 West Main Street, Urbana, IL 61801-2307, USA \\ 3 LIP6, Université Pierre et Marie Curie (Paris VI), 75015 Paris - FRANCE
}

\begin{abstract}
A common feature of congestion control protocols is the presence of information packets used to signal congestion. We study here the question of how frequently such protocols need to generate information packets in order to optimize their performance. Through a number of models, we identify and quantify different types of effects of the frequency of generating information packets. We consider both TCP-type protocols, in which controlling the frequency of information packets is done through static or dynamic delayed ACK options, as well as ATM type flow control, where the optimal time spacing between the generation of network management packets is computed. We show how the spacing between information packets influences the throughput and stability of the system.
\end{abstract}

Keywords: TCP, Delayed ACK, Throughput, Stability, Flow Control.

\section{Introduction}

Congestion and flow control protocols make use of special control packets to indicate to the traffic sources actions to be taken in order to adapt the transmission rates to the available bandwidth. In TCP/IP these are the acknowledgement packets; in the ABR (Available Bit Rate) class of ATM these are the resource management packets. Even when the size of these packets is significantly smaller than that of the data packets, they may still compete over network resources with the data packets and thus decrease the amount of resources available to them. In many cases, they may require an amount of resource much larger than what their size would suggest. For example, when using the IEEE 802.11 MAC protocol, each data as well as ACK packet of the TCP flow requires the same (large) overhead of three link layer packets (RTS, CTS and a link layer ACK). Even in the absence of such overheads, the processing time of an ACK at the destination might require an additional overhead that can be pretty large with respect to the transmission time when very high speed networks are considered. We therefore raise the question of with what frequency flow control protocols should send control packets.

\footnotetext{
* Work of this author was partially supported by the EuroNgi Network of Excellence.

** Research of this author was partially supported by the NSF Grant CCR 00-85917.
} 
We should note that TCP/IP already has the "delayed ACK" option that allows it to reduce the ACK frequency from one ACK for every received packet to one ACK every $d=2$ received packets. Note that ACKs could also be filtered within the network (see e.g. [4] and references therein). However we shall not investigate here the question of how ACKs should be thinned.

We introduce three frameworks in which to study the optimization of the frequency of control packets. The first is the case of TCP/IP traffic sources with routers using drop tail queues. A simple mathematical model is derived for optimizing the amount of ACK thinning at the destination so as to maximize the system's throughput. A simulation study validates the conclusions we obtained from the mathematical model. In the second framework, we study the dynamics of an AIMD (additive increase multiplicative decrease) flow control interacting with a RED type buffer. We model the system's dynamics through a system of delay-differential equations, and study the stability of the system as a function of the frequency of ACKs. We finally propose an abstract model for an optimal rate control with sampled delay information, similar to models used for rate control in the ABR class in ATM [1]. We adopt a linear-quadratic model and optimize the time between two successive packets in information feedback.

\section{A Fixed-Point Method to Model ACK Thinning}

In this section, we use the expression for TCP throughput developed in the literature and standard queueing models to quantify the impact of the delay factor $d$ on the throughput. The throughput $T$ of a TCP connection can be approximated by [7]

$$
\frac{1}{R T T \sqrt{\frac{2 d p}{3}}+R T O \min \left(1,3 \sqrt{\frac{3 d p}{8}}\right) p\left(1+32 p^{2}\right)}
$$

where $p$ is the loss probability of TCP packets, RTT is the round-trip delay experienced by the TCP connection, and RTO is the retransmission timeout.

We start by modeling the network as a bidirectional link. Each direction of the link is modeled by a queue system. Two sets of $N$ symmetric TCP sources send data from both end-points of the link. Each source of the first set connects to a receiver that does not belong to the second set and vice versa. Thus, in each queue TCP packets and ACK packets from different connections are multiplexed in the same queue and served by the same server. Using this model, we assume that losses that occur in the system are only due to congestion, i.e. buffer overflow.

We denote by $\alpha$ the "effective" size of ACK packets and by $Z$ the size of TCP packets. The parameter $\alpha$ would not only model the actual size of ACK packets but also the eventual overheads introduced in the processing time. In order to develop a tractable model, we need a simple formula that relates the throughput at the buffers to the losses that will be experienced there. To that end we shall assume that the packet arrival process at each queue can be approximated by a Poisson process. (We note that the validity of this approximation in a similar 
context has been discussed and examined in [3].) Then, the packet loss probability is the loss probability of an $M / G / 1 / K$ system. Note that the loss probability seen by ACK packets or TCP packets is theoretically the same because of the PASTA property.

Below we propose two modeling approaches for the service time: the exponential service time which provides a simple expression for the losses but gives a rough approximation, and the deterministic service time (whose duration varies according to whether it is an ACK or a data packet) which gives a better approximation but with a more complex expression for the losses. In the deterministic model there are thus two possible values of service times: $\alpha / Z C$ and $1 / C$, where $C$ is the capacity of the link in TCP packets per unit of time.

The Exponential Service Time Case. In this case, the service time of packets is exponentially distributed. The loss probability is then given by the loss probability of an $M / M / 1 / K$ system:

$$
p=\rho^{K} \frac{1-\rho}{1-\rho^{K+1}}
$$

where $\rho$ is the load of the system and it is computed as follows:

$$
\rho=\left(N T+\frac{N T}{d}\right)\left(\frac{1}{d+1} \frac{\alpha}{Z C}+\frac{d}{d+1} \frac{Z}{Z C}\right)=\frac{1}{C}\left(N T+N T \frac{\alpha}{d Z}\right)
$$

We use the fixed-point method to solve numerically the system of equations (1), (2) and (3). The advantage of this simple model is that we can compute the loss probability and thus the throughput for large values of $K$ and $\rho$.

The Deterministic Service Time Case. Here, we modify only the assumption that the service times are exponentially distributed and thus only equation (2) is replaced by the expression of the loss probability of an $M / G / 1 / K$ queue $[8]$ :

$$
p=\frac{1+(\rho-1) f}{1+\rho f}, \text { where } f=\frac{1}{2 \pi i} \oint_{D_{r}} \frac{1}{G(s) s^{K-1}} \mathrm{~d} s
$$

$D_{r}$ is any circle in the complex plane with center 0 and radius $r$ chosen small enough so that all the zeros of the function $G(s)$ are outside $D_{r}$, i.e. $r<|G(z)| \forall z$ such that $G(z)=0$. The complex function $G(s)$ is defined as $G(s)=\operatorname{LST}(b(\lambda(1-s)))-s$ where $L S T(b())$ is the Laplace Stieltjes Transform of the service time distribution $b()$ :

$$
L S T(b(s))=\int_{0}^{\infty} b(t) e^{-s t} d t=\underbrace{\frac{1}{d+1} e^{-(\alpha s / Z C)}}_{A C K}+\underbrace{\frac{d}{d+1} e^{-s / C}}_{T C P}
$$

The parameter $\lambda$ is the total arriving rate at the entrance of each queue, which is equal to $(N T+N T / d)$. Computation of $f$ is detailed in [2].

Again, we solve the three equations (1), (4) and (3) numerically, and we compare the results to those obtained using the exponential time distribution. 
Numerical Results and Simulations. Figure 1 depicts plots of the throughput of TCP as a function of the delay factor $d$. We consider an "effective" size of TCP data packet of 500 bytes and various "effective" sizes of ACKs, ranging between $\alpha=40$ and $\alpha=250$. (as mentioned in the introduction, the difference between actual and effective size of a packet is that additional overhead may be added to its real size due to other protocols of other layers; in addition, processing an ACK at some nodes may take longer than its relative size with respect to a TCP data packet). The queue size is 20 packets and the round-trip time RTT is $200 \mathrm{~ms}$. We set $C$ to $125 \mathrm{TCP}$ packets/s, and $N$ to 1 since the throughput depends only on the fraction $C / N$. The plots were generated numerically using the more precise model of deterministic service times. The figure shows that

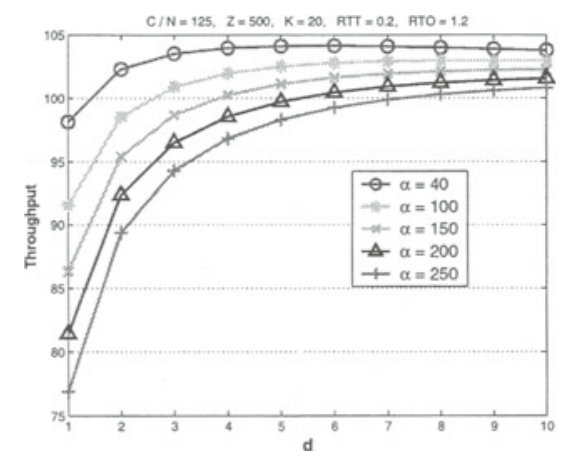

Fig. 1. Effect of $d$ on the throughput for various ACK sizes - the deterministic service time case

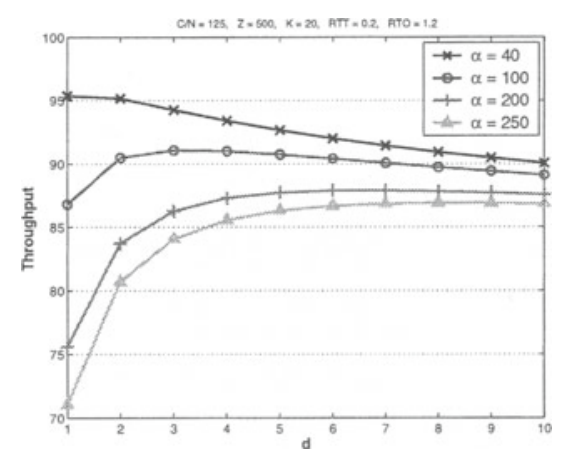

Fig. 2. Effect of $d$ on the throughput for various ACK sizes - the exponential service time case

for the small ACK size 40, spacing the ACK (using $d>1$ ) results in a small improvement of the performance (6\%). However, the throughput is maximized when $d=4$. For higher values of "effective" ACK size $(\alpha \geq 100)$, we see an improvement of $12 \%$ to $31 \%$, with the optimum obtained for example at $d=5$ for $\alpha=100$. Using a value of $d>2$, which is the default value in TCP, results in a relative gain of around $10 \%$. We have observed similar trends with larger link capacities $C$ (larger congestion window) and with much smaller buffer sizes $K$ (higher loss probabilities). When $K \geq 60$, the throughput approaches the value obtained simply by solving $\rho=1$, which gives $T h p t=\frac{C}{N(1+\alpha /(d Z))}$, and the relative gain can reach $50 \%$.

Figure 2 depicts plots of the throughput vs. $d$ with the same parameters as in the previous scenario, but using the exponential service time approximation. In this case, the packet size can represent the average of the "effective" packet size in the network. This model is useful when the packet size in the backbone is variable and the exact distribution is unknown. The figure shows that the trend of the throughput is almost the same, except for the smallest ACK size 40 where spacing the ACK results in deterioration of the performance for all values of 
$d>1$. For larger ACK sizes $(\alpha \geq 200)$, once again, the gain of delaying ACKs goes beyond $20 \%$.

Next, we perform two sets of simulations to study the robustness of the models described herein. First, we study the effect of the number of connections $N$. Then, we look at the effect of the receiver timeout ${ }^{1}$ which is not considered by the two models. We use a similar network configuration as the one described in the model of Section 2, and we add $N$ access links of capacity $1 \mathrm{Mbs}$ each to the bottleneck link. The average round-trip propagation delay $(\approx R T T)$ is set to $200 \mathrm{~ms}$.

In the first set of simulations, we vary the number of connections $N$ from 30 to 200 , and we vary the bottleneck capacity $C$ in order to keep the ratio $C / N$ constant at 125 . We fix the ACK size to $\alpha=100$. In this set of simulations, we use the default value of the receiver timeout which is set to $100 \mathrm{~ms}$. Figure 3 plots the average TCP throughput vs. the delayed factor $d$, using the two models and simulation traces for $N=30,50,100$ and 200 .

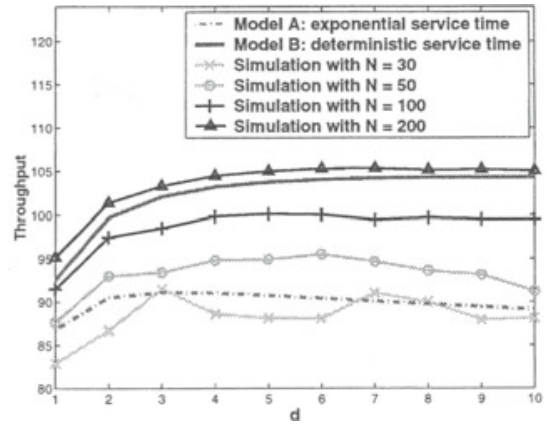

Fig. 3. Simulation results vs. numerical results

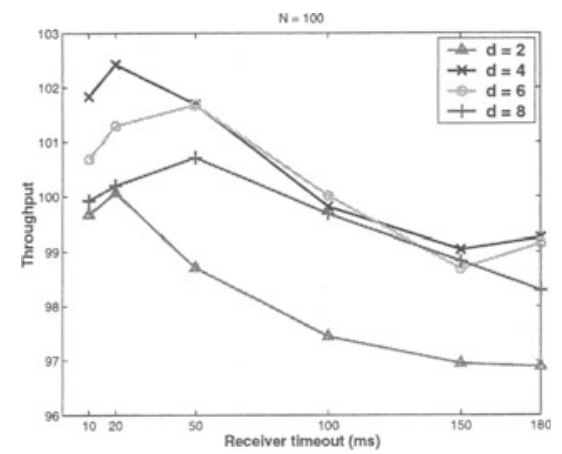

Fig. 4. Effect of the receiver timeout on the TCP throughput

The main observation we make is that as $N$ increases, the relative error induced by the deterministic model decreases notably. This is because the resulting process of multiplexing the TCP connections approaches a Poisson process [3].

Next, we examine the impact of receiver timeout on TCP throughput. We use the same parameter values as in the previous simulations, and set $N=100$. We vary the receiver timeout from $t_{1}=10 \mathrm{~ms}$ to $t_{2}=180 \mathrm{~ms}$. Note that $t_{1}$ is just larger than the minimum inter-arrival time of two consecutive TCP packets sent in the same window, and $t_{2}$ is just less than RTT. Figure 4 depicts plots of the average TCP throughput as a function of the receiver timeout for various values of $d$. We see clearly that when the timeout is large, then the throughput

\footnotetext{
${ }^{1}$ The receiver timeout is used to send an ACK for arriving packets even before $d$ packets arrived at the receiver if the time since the unacknowledged packet arrived exceeds the timeout.
} 
is reduced. In the cases $d=2$ and $d=4$, the throughput is maximized when the timer is equal to $20 \mathrm{~ms}$. For $d=6$ and $d=8$, the throughput is maximized at a timer of $50 \mathrm{~ms}$.

Here, the gain in the throughput is relatively small $(\approx 3 \%)$, but this is because the average window size is also small $(\approx 20)$. For larger window sizes, for example when the delay-bandwidth product is large, the gain is more significant. However, setting a small value for the timeout is risky since the packet inter-arrival time depends on the cross traffic along the path and hence could vary over time.

More generally, it is difficult to find a constant timeout that is adequate for all network scenarios. Another alternative is to compute dynamically the timeout using a similar method as the one used by the sender to compute the retransmission timeout.

To summarize, it is worth using a delay factor $d>2$, particularly for long TCP connections and when the window size is large. In fact, the improvement obtained from reducing the number of ACKs in the network is more significant than the decrease in the throughput due to the lack of information update.

\section{Control Model of TCP Traversing a RED Buffer}

After focusing on the quantitative impact of the rate of information on the throughput, we next study how stability conditions are influenced by the rate of information packets. We assume that $N$ symmetric persistent TCP connections share a bottleneck link of capacity $C$ located close to the source, assumed to operate in the congestion avoidance regime. Let $W(t)$ be the window size of a connection at time $t$, and $R$ the round trip delay (including queueing delay), which is assumed to be a constant (this assumption holds when queueing delays are much smaller than propagation delays). Let $p(t)$ be the loss (or marking) probability of the RED buffer at time $t$. Let $q(t)$ be the amount of buffered traffic at the queue at the bottleneck link. We consider a fluid approximation of the window size of TCP given by $\frac{\mathrm{d} W}{\mathrm{~d} t}=\frac{1}{R}-\frac{W(t) W(t-R)}{2 R} p(t-R)$, see [6]. This equation is obtained under the assumptions that (i) the delayed ACK mechanism is not used; and (ii) a new ACK is generated with each TCP packet that arrives at the destination, resulting in an increase of the window size by one unit every round trip time.

We shall now consider the possibility of using a (dynamic) delayed ACK approach in which an ACK is generated for every $d$ TCP packets that arrive at the destination. $d$ will be considered as a control variable and will thus be allowed to be time dependent. Both the increase rate as well as the decrease rate are divided by a factor $d$ since the rate of ACKs that arrive at the sources is $d$ times smaller. In particular indications for decreasing the rate (we assume that ACKs have marks indicating congestion) return less frequently. The window size evolution then becomes

$$
\frac{\mathrm{d} W}{\mathrm{~d} t}=\frac{1}{R d(t-R)}-\frac{W(t) W(t-R)}{2 R d(t-R)} p(t-R) .
$$


The queue dynamics are given in [6] by $\frac{\mathrm{d} q}{\mathrm{~d} t}=\frac{N}{R} W(t)-C$. We shall assume that not only the TCP packets have to queue but also the ACKs. To model the difference between the size of an ACK and that of a TCP packet, we assume that an ACK requires a fraction $\gamma$ of buffer space required by a TCP packet. Under this assumption, the queue dynamics above are modified to $\frac{\mathrm{d} q}{\mathrm{~d} t}=\frac{N}{R} W(t)-$ $C+\frac{\gamma W(t-R) N}{d(t-R) R}$. The tradeoff that influences the choice of the control $d$ will be the following: on one hand, when the window size is small, we may want $d$ to be small so that the window size can grow quickly so as to achieve higher throughput. On the other hand, when the window size is large then we may want to increase $d$ so as to limit the congestion due to ACKs in the bottleneck queue. We shall analyze in this section a linear control mechanism in which $d$ has the form $d(t)=\alpha(1+\beta W(t)), \quad \alpha>0, \beta \geq 0$. Finally, we shall consider the RED buffer marking probability (ignoring the averaging of the queue size) in its linear operation regime: $p(t)=\eta_{1} q(t)-\eta_{2}$. We now summarize the system's overall dynamics below:

$$
\begin{aligned}
\frac{\mathrm{d} W}{\mathrm{~d} t} & =\frac{1}{R d(t-R)}-\frac{W(t) W(t-R)}{2 R d(t-R)} p(t-R) \\
\frac{\mathrm{d} q}{\mathrm{~d} t} & =\frac{N}{R} W(t)-C+\frac{\gamma W(t-R) N}{d(t-R) R} \\
d(t) & =\alpha(1+\beta W(t)), \quad p(t)=\eta_{1} q(t)-\eta_{2}
\end{aligned}
$$

Equilibrium: The equilibrium point is obtained by equating to 0 the time derivatives in the previous equations. This leads to:

$$
\begin{aligned}
& \frac{N}{R} W_{o}+\frac{N \gamma W_{o}}{d_{o} R}=C \Rightarrow W_{o}=\frac{C R}{N\left(1+\gamma / d_{o}\right)} \\
& d_{o}=\alpha\left(1+\beta W_{o}\right), \quad W_{o}^{2} p_{o}=2 \Rightarrow p_{o}=2 / W_{o}^{2} \\
& p_{o}=\eta_{1} q_{o}-\eta_{2} \Rightarrow q_{o}=\left(p_{o}+\eta_{2}\right) / \eta_{1}
\end{aligned}
$$

Hence the throughput is given by: $T h p=\frac{W_{o}}{R}=\frac{C}{N\left(1+\gamma / d_{o}\right)}$. The throughput is seen to increase with $d_{o}$.

A linearization of the dynamical system in a neighborhood of the equilibrium point yields

$$
\begin{aligned}
\frac{\mathrm{d} \delta W}{\mathrm{~d} t} & =-\frac{1}{R W_{o} d_{o}} \delta W(t)-\frac{1}{R W_{o} d_{o}} \delta W(t-R)-\frac{\eta_{1} W_{o}^{2}}{2 R d_{o}} \delta q(t-R) \\
\frac{\mathrm{d} \delta q}{\mathrm{~d} t} & =\frac{N}{R} \delta W(t)+\frac{\alpha \gamma N}{d_{o}^{2} R} \delta W(t-R),
\end{aligned}
$$

where $\delta$ stands for the shifted version of variables in which the equilibrium value is subtracted (e.g. $\delta W:=W-W_{o}$ ). Taking the Laplace transform of these equations we obtain

$$
\begin{aligned}
s \delta W(s) & =-\frac{1}{R W_{o} d_{o}} \delta W(s)-\frac{e^{-s R}}{R W_{o} d_{o}} \delta W(s)-\frac{\eta_{1} W_{o}^{2}}{2 R d_{o}} e^{-s R} \delta q(s) \\
s \delta q(s) & =\left(\frac{N}{R}+\frac{\alpha \gamma N}{d_{o}^{2} R} e^{-s R}\right) \delta W(s)
\end{aligned}
$$


With $z=s R$, the stability condition is then given by requiring that all zeros of $g(z)=0$ have strictly negative real parts, where $g(z)$ is given by

$$
g(z)=z^{2}+\frac{z}{W_{o} d_{o}}\left(1+e^{-z}\right)+\frac{\eta_{1} W_{o}^{2} N}{2 d_{o}}\left(1+\frac{\alpha \gamma}{d_{o}^{2}} e^{-z}\right) e^{-z}
$$

The goal. One may identify two possible goals: (i) maximize the system throughput while keeping it stable, and (ii) for a given desired throughput, make the system "as stable as possible" by which we mean to choose the parameters so as to have the real part of the largest zero of $g(z)$ as negative as possible.

Note that whereas the throughput only depends on $d_{o}, \gamma, N$ and $C$, and not directly on the values of $\alpha, \beta, \eta_{1}, \eta_{2}$, the stability regime does depend directly on $\alpha$ and $\eta_{1}$, and hence these parameters do enter into the optimization under goal (i). For the second goal, we may first compute $d_{o}$ and then optimize stability with respect to the other parameters.

A numerical example. This numerical example is picked to show that the system could be unstable for meaningful choices of the values of the parameters: $\eta_{1}=0.001, \eta_{2}=0.02, \alpha=1, d_{0}=3$. Further let $N=5, \gamma=250 / 500=$ $0.5, R C=100$ so that $R C>>q_{o} . W_{o}=\frac{C R}{N\left(1+\gamma / d_{o}\right)}=100 /(5 \times 1.166)=$ 17.142857. Hence $p_{o}=2 / W_{o}^{2}=0.006806, \beta=\left(d_{0} / \alpha-1\right) / W_{o}=0.11666$ and $q_{0}=\frac{p_{0}+\eta_{2}}{\eta_{1}}=26.80555$, which is indeed small with respect to $R C$. We can verify that $z=0.09964430039+0.4704656808 * I$ is a zero of $(9)$, and hence the system is unstable.

Using the Model: Impact on Stability. Examining the form of $g(z)=0$ (whose solutions provide the stability condition), we observe the following:

- The stability condition is not a function of the round trip delay. This is seen directly from (6)-(8): if we scale time so that a time unit corresponds to a round trip time, we arrive at a new system of equations which do not involve $R$.

- From the form of the expression $g(z)$ we see that for the same values of $C, R, N, \gamma, \eta_{1}, \eta_{2}$ and for the same value $d_{o}$ at equilibrium, the stability region can change according to the choice of the parameters $\alpha$ and $\beta$. $\beta=0$ corresponds to a non-dynamic value of $d$ (i.e. a value that does not change with $W$ ) in which case $\alpha=d_{o}$.

- We also see that for fixed $C, R, N, \gamma, \alpha, \beta$ and a fixed queue size $q_{o}$, at equilibrium, $g(z)$ will be influenced by $\eta_{1}$ (and hence the stability region). In fact $g(z)$ does not depend on $\eta_{2}$, but note that since we assume that $R$ includes the mean queueing delay (which is proportional to $q_{o}$ ), this means that fixing $q_{o}$ and $\eta_{1}$ already determines $\eta_{2}$.

In the following, we choose a scenario that shows clearly the impact of using a dynamic delayed ACK factor $d$ on the stability of RED. We fix the parameters used in the numerical example. Then, we set $N=14$, and vary $\alpha$ from 1 to 4 . Since we fix $d_{0}, \beta$ is deduced. Table 1 presents the results concerning the stability of the system. 
Table 1. Impact of dynamic control on stability

\begin{tabular}{|c|c|c|c|}
\hline & $\alpha$ & $\Rightarrow \beta$ & The system is \\
\hline 3 & & $0.33(W \nearrow d \nearrow)$ & stable \\
\hline 3 & 2 & $0.08(W \nearrow d \nearrow)$ & stable \\
\hline 3 & 3 & 0 (no control) & unstable \\
\hline 3 & & $-0.04(W \nearrow d \searrow)$ & unstable \\
\hline
\end{tabular}

The first observation we make is that when $\alpha=3\left(=d_{0}\right)$, the system is unstable, which means that when there is no dynamic variation of the delayed factor $d$, the system is unstable. However, for $\alpha=1$ or 2 , the system is stable. Besides, $\beta$ is positive, which means that the linear control is correct $(d(t)$ is a non-decreasing function of $W(t))$. When $\alpha=4, \beta$ is negative and the system is unstable. These results corroborate the fact that with an increase of the TCP window, we should increase the delay factor $d$. Moreover, using an adequate adaptive control of $d$, we can improve the stability of the system.

\section{Linear-Quadratic Approaches to Flow Control}

We now study the problem of optimum choice of the time interval between successive transmission of information using a control theoretic approach within a linear-quadratic framework. Such frameworks have frequently been used for approximating rate-based flow control by ignoring the nonlinearity at queue boundaries (empty or full buffers). More precisely, we make four simplifying assumptions:

1. Fluid approximation. We replace a discrete number of packets by a continuous fluid.

2. Linearized dynamics. The network has linearized dynamics for the control of queue length; see (10) below: we neglect losses when the buffer is full, and we neglect the boundary effect of an empty queue. As discussed below, we set some desirable threshold on the queue length which we attempt to track as closely as possible so as to avoid large queues (which might lead to losses) or empty queues (which might result in loss of potential throughput).

3. Bottleneck assumption. All performance measures (such as throughput, delays, loss probabilities, etc.) are determined essentially by a bottleneck node.

4. Information flow. Information on the queue length is sent to the controller periodically, every $1 / \lambda$ seconds.

We now introduce the model. Let $q(t)$ denote the queue length at a bottleneck link. Assume that the information packets use the same link and have priority over data packets. The link capacity available to data packets, $C$, is thus assumed to depend on $\lambda: C(\lambda)=C-\frac{a}{\lambda}$. We assume that other uncontrolled inputs share the buffer, and their total input rate is given by $C_{1}+v(t)$ where $C_{1}$ is some constant and $v$ represents a stochastic process with zero mean. The average rate of the controlled source is assumed to be $C_{2}=C-C_{1}$. Thus we let $u(t)+C_{2}$ be 
the rate of the controlled input into the queue at time $t$. Then the queue length dynamics are given by

$$
d q=u \mathrm{~d} t+\mathrm{d} v
$$

which is idealized because the end-point effects have been ignored. The objectives of the flow controller are (i) to ensure that the bottleneck queue size stays around some desired level $\bar{Q}$, and (ii) to minimize variations in the rates. The choice of $\bar{Q}$ and the variability around it have a direct impact on loss probabilities and throughput. We therefore define a shifted version of $q: x(t):=q(t)-\bar{Q}$, in view of which (10) now becomes $d x=u \mathrm{~d} t+\mathrm{d} v$. An appropriate local cost function that is compatible with the objectives stated above would be the one that penalizes variations in $x(t)$ and $u(t)$ around zero - a candidate for which is the weighted quadratic cost function: $x^{2}+k u^{2}$. We seek an optimal control policy among those which choose $u(t)$ as a function of the queue length at the times when information is available: $u(t)=\mu(x(0), x(\lambda), \ldots, x(n \lambda))$, for $t \in[n \lambda,(n+1) \lambda), \quad n=0,1,2, \ldots$

LQG model. We assume that $v$ is a zero-mean Brownian motion with incremental variance $r$. The expected average cost for a given policy $\mu$ and initial state $x$ is defined as $J(x, \mu, \lambda)=\lim _{T \rightarrow \infty} \frac{1}{T} E_{x}^{\mu}\left[\int_{0}^{T}\left(x^{2}(t)+k u^{2}(t)\right) d t\right]$ where $E_{x}^{\mu}$ is the expectation with respect to the probability measure induced by a policy $\mu$ and an initial state $x$. We first seek to obtain the optimal policy and value for a given $\lambda: J(x, \lambda):=\min _{\mu} J(x, \mu, \lambda)$.

Theorem 1. [2] The optimal value of the LQG problem is independent of the initial state $x$ and is given by $J(\lambda)=\frac{\lambda^{2}}{2} r+\sqrt{k} r$, and the unique policy that attains the minimum is given by

$$
u^{*}(t)=\mu^{*}(x)=-\frac{1}{\sqrt{k}} \exp \left(-\frac{t-n \lambda}{\sqrt{k}}\right) x(n \lambda) \text { for } t \in[n \lambda,(n+1) \lambda) .
$$

We thus conclude that as long as $\lambda^{2}$ is much smaller than $\sqrt{k}$, the value is quite insensitive to changes in the spacing $\lambda$. On the other hand, when it is much larger than $\sqrt{k}$, we see that the spacing of information packets has a huge impact on the performance: the cost grows quadratically in the spacing.

Optimal spacing of information for the LQG model. We obtained above the optimal control policy for a given parameter $\lambda$ of spacing of information packets. Our next goal is to optimize $\lambda$. In addition to the cost $J(\lambda)$ which we have obtained, we assume that the flow control has some utility $U(\lambda)$ that can represent the utility for the average throughput $C-a / \lambda$ available for the data packets. We shall consider

1. A utility linear in the average throughput: $U(\lambda)=C-a / \lambda$.

2. A logarithmic utility: $U(\lambda)=\log (C-a / \lambda)$.

The cost to be minimized in both cases is $Z(\lambda)=J(\lambda)-\gamma U(\lambda)$. Straightforward minimization leads to the following: 
Theorem 2. The optimal information spacing is given by

(i) linear case: $\lambda^{*}=\left(\frac{\gamma a}{r}\right)^{1 / 3}$ (ii) logarithmic case:

$$
\begin{aligned}
\lambda^{*} & =\frac{1}{6}\left(108 \beta+8 \alpha^{3}+12 \sqrt{81 \beta^{2}+12 \beta \alpha^{3}}\right)^{1 / 3}+\frac{\alpha}{3} \\
& +\frac{2 \alpha^{2}}{3\left(108 \beta+8 \alpha^{3}+12 \sqrt{81 \beta^{2}+12 \beta \alpha^{3}}\right)^{1 / 3}} \text { where } \alpha=\frac{a}{C} \text { and } \beta=\frac{\gamma a}{r C} .
\end{aligned}
$$

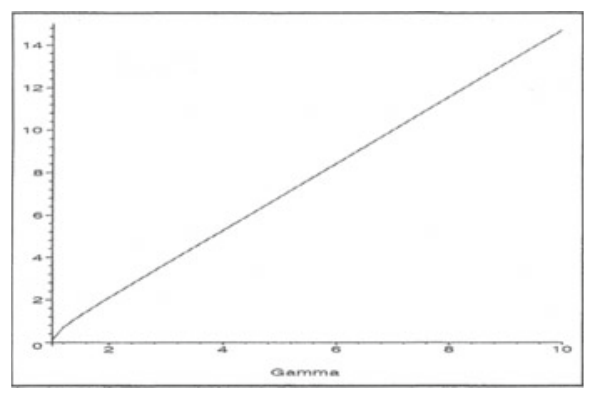

Fig. 5. Maximum spacing as a function of the attenuation $\Gamma$

An $H^{\infty}$ approach. In this approach we do not make any statistical assumptions on the distribution of the noise process, and we adopt instead a robust approach that guarantees the best performance under the worst-case conditions. More precisely, we introduce

$$
V(\mu, \lambda)=\sup _{\left\{v_{n}\right\}_{n=-\infty}^{\infty}} \frac{L(\mu, v, \lambda)}{\|v\|^{2}} \text { where } L(\mu, \nu, \lambda)=\int_{-\infty}^{\infty}\left(x^{2}(t)+k u^{2}(t)\right) d t
$$

and $\|v\|$ is the $L^{2}$ norm of $v:\|v\|=\sqrt{\sum_{n=-\infty}^{\infty}\left(v_{n}\right)^{2}}$. We seek a $\mu$ that minimizes $V(\mu, \lambda)$; denote its infimum over $\mu$ by $\left(\Gamma^{*}\right)^{2}$.

Define a soft-constrained cost function $L_{\Gamma}(\mu, v, \lambda):=L(\mu, v, \lambda)-\Gamma^{2}\|v\|^{2}$, and consider a two player game where $L_{\Gamma}$ is to be minimized by Player 1 (controlling $\mu$ ) and maximized by Player 2 (controlling $v$ ). If there exists some policy $\mu^{*}$ for the problem of minimizing $L(\mu, \lambda)$, then [5]: $\sup _{v} L_{\Gamma^{*}}\left(\mu^{*}, v, \lambda\right)=$ $\inf _{\mu} \sup _{v} L_{\Gamma^{*}}(\mu, v, \lambda)$. The quantity above is the upper value of the zero-sum game with kernel $L_{\Gamma^{*}}$, which in fact equals zero. It can be shown that for any $\Gamma \geq \Gamma^{*}$, the upper value of the game with parameterized kernel $L_{\Gamma}$ is zero, and for $\Gamma<\Gamma^{*}$, its upper value is infinite. Hence, $\Gamma^{*}$ is the smallest positive scalar $\Gamma$ for which the zero-sum game with kernel $L_{\Gamma}$ has a finite upper value. Instead of obtaining $\mu^{*}$ defined above, we will solve a parameterized class of controllers, $\left\{\mu^{\Gamma}, \Gamma>\Gamma^{*}\right\}$, where $\mu^{\Gamma}$ is obtained from $\sup _{v} L_{\Gamma}\left(\mu^{\Gamma}, v, \lambda\right)=\inf _{f} \sup _{v} L_{\Gamma}(\mu, v, \lambda)$. The controller $\mu^{\Gamma}$ will clearly have 
the property that it ensures a performance level $\Gamma^{2}$ for the index adopted in (12), i.e. the attenuation is bounded by $\Gamma$, i.e., $V\left(\mu^{\Gamma}, \lambda\right) \leq \Gamma^{2}$. It turns out that

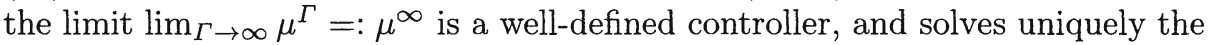
control problem with the previous Gaussian model. Note that $\Gamma^{*}$ will be a function of $\lambda$. Our goal is to determine the largest value of spacing for which there exists a policy that guarantees a given level of attenuation.

Theorem 3. For a given level of attenuation $\Gamma$, the largest spacing $\lambda$ of information for which there is a policy $\mu^{\Gamma}$ such that $V\left(\mu^{\Gamma}, \lambda\right) \leq \Gamma^{2}$ is given by, for $\Gamma>k, \lambda=\frac{\pi}{2} \Gamma-\Gamma \arctan \frac{1}{\sqrt{\Gamma^{2} / k-1}}$.

The proof follows from the material in [5, Chapter 5.3]. Figure 5 shows how the spacing grows as a function of the desired achievable attenuation for $k=1$. The spacing ( $y$-axis) is seen to be concave increasing in the desired attenuation level, and asymptotically its growth is linear in $\Gamma$.

\section{Conclusions}

We have studied various facets of the problem of determining how frequently information should be sent to the source in congestion control mechanisms. We have shown that the rate of information can have an impact on the throughput of the system, as conveying information requires resources that are then not available for data packet transmission. We have further identified an impact on the stability of congestion control. Finally, we have formulated two dynamic optimization (control) problems, one using a stochastic model for queue dynamics, and the other one using a deterministic worst-case model. In both cases, we have obtained apparent tradeoffs dictating the optimal choice of the spacing between successive information packets.

\section{References}

1. E. Altman and T. Başar, "Multi-user rate-based flow control", IEEE Trans. on Communications, pp. 940-949, 1998.

2. E. Altman, T. Başar and N. Malouch, "The Role of Information Update in Flow Control", INRIA Research report, 2004.

3. U. Ayesta, K. Avrachenkov, E. Altman, C. Barakat, P. Dube, "Simulation Analysis and Fixed Point Approach for Multiplexed TCP flows", PFLDnet'03: Workshop on Protocols for Fast Long-Distance Networks, CERN-Geneva, Switzerland, 2003.

4. C. Barakat and E. Altman, "On ACK filtering on a slow reverse channel", QOFIS, Berlin, Germany, September 2000.

5. T. Başar and P. Bernhard, $H^{\infty}$-Optimal Control and Relaxed Minimax Design Problems: A Dynamic Game Approach, Birkhäuser, Boston, MA, 1995 (2nd edt).

6. C. Hollot, V. Misra, D. Towsley and W.-B. Gong, "A control theoretic analysis of RED" CMPSCI Technical Report TR 00-41, July 2000.

7. J. Padhye, V. Firoiu, D. Towsley and J. Kurose, "Modeling TCP throughput: A simple model and its empirical validation", ACM SIGCOMM'98, 1998.

8. J. W. Cohen "The Single Server Queue", North Holland publishing company, 1982. 